\title{
COMPOSIÇÃO QUÍMICA E ATIVIDADES BIOLÓGICAS DAS FOLHAS DE Cynara scolymus L. (ALCACHOFRA) CULTIVADA NO BRASIL
}

Vânia Floriani Noldin e Valdir Cechinel Filho*

Núcleo de Investigações Químico-Farmacêuticas, Universidade do Vale do Itajaí, CP 360, 88302-202 Itajaí - SC

Franco Delle Monache

Centro Chimica Recettori, Istituto di Chimica, Universitá Cattolica del Sacro Cuore, Largo F Vito 1, Roma-Itália

Jean Carlo Benassi, Irma Luiza Christmann e Rozangela Curi Pedrosa

Departamento de Bioquímica, Universidade Federal de Santa Catarina, CP 246, 88040-900 Florianópolis - SC

Rosendo Augusto Yunes

Departamento de Química, Universidade Federal de Santa Catarina, CP 476, 88040-900 Florianópolis - SC

Recebido em 16/4/02; aceito em 8/10/02

CHEMICAL COMPOSITION AND BIOLOGICAL ACTIVITIES OF THE LEAVES OF Cynara scolymus L. (ARTICHOKE) CULTIVATED IN BRAZIL. The present paper describes the chemical composition and biological activities of artichoke cultivated in Brazil. Our studies demonstrated that glycosyl flavonoids (cynaroside and scolymoside), are the major constituents, along with cynaropicrin, a sesquiterpene lactone, and the triterpene lupeol. Cynarin, which is the main compound described for artichoke, was detected in very low concentration. Hexanic fraction exhibited considerable cytotoxicity and diuretic activities.

Keywords: Cynara scolymus; flavonoids; diuretic.

\section{INTRODUÇÃO}

A utilização de plantas medicinais tornou-se um recurso terapêutico alternativo de grande aceitação pela população e vem crescendo junto a comunidade médica, desde que sejam utilizadas plantas cujas atividades biológicas tenham sido investigadas cientificamente, comprovando sua eficácia e segurança ${ }^{1,2}$.

A importância das plantas medicinais deve-se também por sua contribuição como fonte natural de fármacos e por proporcionar grandes chances de obter-se uma molécula protótipo devido à diversidade de constituintes presentes nestas ${ }^{2-5}$. No entanto, inúmeras plantas que são usadas em preparações fitoterápicas carecem de um maior controle de qualidade, uma vez que a literatura científica indica que muitas destas podem apresentar substâncias tóxicas ou composição química variável ${ }^{6,7}$.

Assim, o presente estudo teve como objetivos investigar a composição química e os efeitos biológicos da Cynara scolymus Linné, cultivada no Brasil. Esta planta é conhecida popularmente como alcachofra, pertencente à família Compositae, que compreende cerca de 920 gêneros com aproximadamente 19 mil espécies, cujos hábitos são muito variados ${ }^{8}$. A C. scolymus é oriunda do Mediterrâneo, sendo que seu cultivo se dá por sementes e está difundido mundialmente, já que é utilizada para fins medicinais e alimentícios.

Os principais componentes químicos presentes nas folhas da alcachofra são os ácidos fenólicos, flavonóides e sesquiterpenos. A cinarina (1) (ácido monocafeioilquínico) é relatada como princípio ativo da planta ${ }^{9,10}$. Vários estudos biológicos com extratos brutos e purificados de alcachofra, realizados tanto em animais quanto em humanos, demonstraram atividades hipolipidêmica, hepatoprotetora, colerética, colagoga, antioxidante e outras ${ }^{11-13}$.

Cabe ressaltar, porém, que os estudos encontrados na literatura são abordados com plantas cultivadas na Europa. Considerando a variabilidade da composição química devido a vários fatores (ambien-

*e-mail: cechinel@univali.br tais, sazonais, etc), optou-se em investigar a composição química da alcachofra cultivada no Brasil e que está presente em várias preparações fitoterápicas.

\section{PARTE EXPERIMENTAL}

\section{Coleta do material e preparação dos extratos}

Utilizou-se folhas da C. scolymus, oriundas da Central de Plantas em Curitiba, coletadas em fevereiro de 2000, as quais foram devidamente identificadas e posteriormente secas e moídas. Este material $(1900 \mathrm{~g})$ foi macerado estaticamente por sete dias com metanol, sendo, em seguida, concentrado, usando-se evaporador rotatório sob pressão reduzida, para obtenção do extrato metanólico bruto $(\mathrm{EMB})^{2}$. Este foi particionado com solventes de polaridade crescente, obtendo-se as seguintes frações e seus respectivos rendimentos: Hexano $(0,16 \%)$, Diclorometano (DCM) (1\%), Acetato de Etila (AE) $(0,29 \%)$ e Butanol $(0,65 \%)$. Estas frações foram cromatografadas separadamente em coluna de sílica gel, monitoradas por cromatografia em camada delgada. A fração hexânica (1 g), eluída continuamente com hexano/acetona na proporção de 95:5 permitiu a obtenção de $29 \mathrm{mg}$ de um sólido branco, identificado como lupeol (2), cujos dados de IV e RMN ( $\left.{ }^{1} \mathrm{H} \mathrm{e}{ }^{13} \mathrm{C}\right)$ são idênticos aos da literatura ${ }^{14}$. Sua estrutura foi confirmada por Co-CCD com uma amostra autêntica adquirida comercialmente. A fração diclorometânica (5 g), quando submetida à CC e eluída com hexano/acetona com aumento gradativo de polaridade, possibilitou a obtenção de $280 \mathrm{mg}$ de um composto amareloclaro, de aspecto oleoso, identificado como $\alpha$-metilenobutirolactona, uma lactona sesquiterpênica, denominada de cinaropicrina (3). Os dados espectroscópicos foram comparados com aqueles da literatu$\mathrm{ra}^{15}$. Durante o particionamento da fração AE, ocorreu a precipitação de $130 \mathrm{mg}$ de um composto de coloração amarela, o qual foi identificado como o flavonóide cinarosídio (luteolina-7-O-glicosídio) (4). Os dados de IV, RMN- ${ }^{1} \mathrm{H}$ e RMN- ${ }^{13} \mathrm{C}$ são similares à literatura ${ }^{16}$. Esta fração ( $1 \mathrm{~g})$ foi cromatografada em coluna aberta de sílica gel e 
eluída com clorofórmio/metanol. As subfrações que apresentaram perfil similar por CCD foram reunidas e recromatografadas em $\mathrm{CC}$ / Sephadex LH-20, fornecendo mais $7 \mathrm{mg}$ de cinarosídio (4) e $3 \mathrm{mg}$ de ácido cafeico (5), identificado através de comparação direta com uma amostra autêntica adquirida comercialmente.

A fração butanólica (5 g) cromatografada em CC de sílica gel, eluída com clorofórmio/metanol em diferentes proporções, rendeu quatro subfrações. Estas foram cromatografadas em CC usando Sephadex LH-20 como fase estacionária e metanol:água 9:1 como fase móvel, obtendo-se $9 \mathrm{mg}$ do flavonóide escolimosídio (luteolina7-O-rutinosídio) (6), e somente traços de cinarina (1) e ácido clorogênico (7), que foram identificados apenas por Co-CCD, comparados com amostras autênticas destes compostos.

\section{Ensaios biológicos}

\section{Toxicidade sobre Artemia salina}

Os ensaios de toxicidade foram realizados conforme o método de Meyer $^{17}$, onde $2 \mathrm{mg} / \mathrm{ml}$ dos extratos hexânico, diclorometano, butanólico, acetato de etila, e também dos compostos cinarosídio (4) e cinaropicrina (3) e do controle positivo (sulfato de quinidina), preparados em solução aquosa de sal marinho sintético $(38 \mathrm{~g} / \mathrm{L})$ com $1 \%$ DMSO (v/v), foram incubados em placas "multiwell" durante 48 h com Artemia salina $(n=10)$ a $30^{\circ} \mathrm{C}$. Após incubação, foi feita contagem do número de microcrustáceos mortos e calculada a percentagem de mortalidade. Os extratos são geralmente considerados ativos quando o Brine Shrimp Test (BST) for menor que $1000 \mathrm{ppm}^{17}$.

\section{Atividade diurética}

Para a determinação da atividade diurética foi seguido o modelo descrito por Kau e colaboradores ${ }^{18}$, onde ratos machos $(n=6,250 \pm$ $15 \mathrm{~g}$ ) foram pré-tratados com uma solução de salina $(50 \mathrm{~mL})$ via oral, e uma hora após distribuídos em 3 diferentes grupos: grupo controle negativo (tratado com água destilada), grupo controle positivo (tratado com hidroclorotiazida, $25 \mathrm{mg} / \mathrm{kg}$ de peso) e o grupo teste (tratado com $200 \mathrm{mg} / \mathrm{kg}$ de peso com extratos hexânico e metanólico obtidos da C. scolymus). Os animais foram mantidos em gaiolas metabólicas e o volume de urina medido a cada $2 \mathrm{~h}$ durante $8 \mathrm{~h}$. Após este período, o volume final foi anotado e, posteriormente, determinados os íons de sódio, potássio e cloro de cada amostra por fotometria de chama. Os resultados obtidos foram avaliados estatisticamente utilizando teste ANOVA complementado por Tukey-Kramer ${ }^{18}$, admitindo-se um nível de significância $\mathrm{p}<0,01$.

\section{RESULTADOS E DISCUSSÃO}

A partir do extrato metanólico bruto das folhas da C. scolymus obteve-se, através de partições sucessivas, as seguintes frações: fração hexânica, fração DCM, fração AE e fração butanólica. Da fração hexânica, obteve-se o triterpeno lupeol (2), que ocorre naturalmente em muitas plantas, principalmente nas flores da família Compositae, o qual apresenta várias ações farmacológicas ${ }^{19,20}$.

A planta Dendropanax cf. querceti, na qual o lupeol é o composto ativo, demonstrou atividade citotóxica contra células tumorais HepG2, A-431 e H-4IIE, cujo mecanismo de ação é a inibição da enzima topoisomerase II. Em outros estudos, o lupeol demonstrou atividade sobre a proliferação de queratinócitos na pele, atividades nefro-protetoras tanto na exposição crônica ao cádmio, quanto na redução da excreção de oxalato em determinadas patologias e que causaria danos no túbulo renal. Atividades anti-tumorais, antioxidantes e liberadores de mediadores da resposta imunológica também são efei- tos farmacológicos deste composto ${ }^{21-26}$.

A cinaropicrina (3), isolada da fração diclorometânica com considerável rendimento (5\% a partir da fração diclorometânica), está presente em várias espécies da família Compositae, as quais são ricas em lactonas sesquiterpênicas, geralmente caracterizadas por conterem uma função $\alpha$-metileno- $\gamma$-lactona, que reage com grupos sulfidrilas e com resíduos de cisteína de aminoácidos, podendo ser responsáveis por eventuais ações tóxicas, já que ocorre naturalmente e encontra-se distribuída em várias plantas ${ }^{27}$. Este composto isolado do extrato de Saussurea Lappa radix (Compositae) mostrou ter propriedades inibitórias contra a produção do fator de necrose tumoral (TNF- $\alpha$ ), o qual está envolvido em processos inflamatórios. A eficácia da cinaropicrina foi comparada com o fármaco prednisolona, potente antiinflamatório glicocorticóide ${ }^{28,29}$. Outros autores atribuem que a cinaropicrina, além de propriedades antitumorais, antimicrobianas e antifúngicas, eventualmente podem causar dermatite alérgica, inibição da secreção de plaquetas e efeitos citotóxicos ${ }^{9,27,28,30,31}$.

A fração AE permitiu o isolamento do flavonóide cinarosídio (4) e do ácido cafeico (5), este último presente em baixa concentração na planta. A fração butanólica possibilitou o isolamento de flavonóide escolimosídio (6). Ao contrário do que demonstra a literatura para a alcachofra cultivada na Europa, nossos estudos indicaram apenas traços de cinarina (1) e ácido clorogênico (7), sendo o primeiro considerado um marcador químico e biológico para a planta em questão.

Os flavonóides obtidos das frações acetato de etila e butanol apresentam várias ações farmacológicas, tais como atividades antibacteriana, antiinflamatória, antioxidante, hipolipidêmica e outras ${ }^{9,12}$.
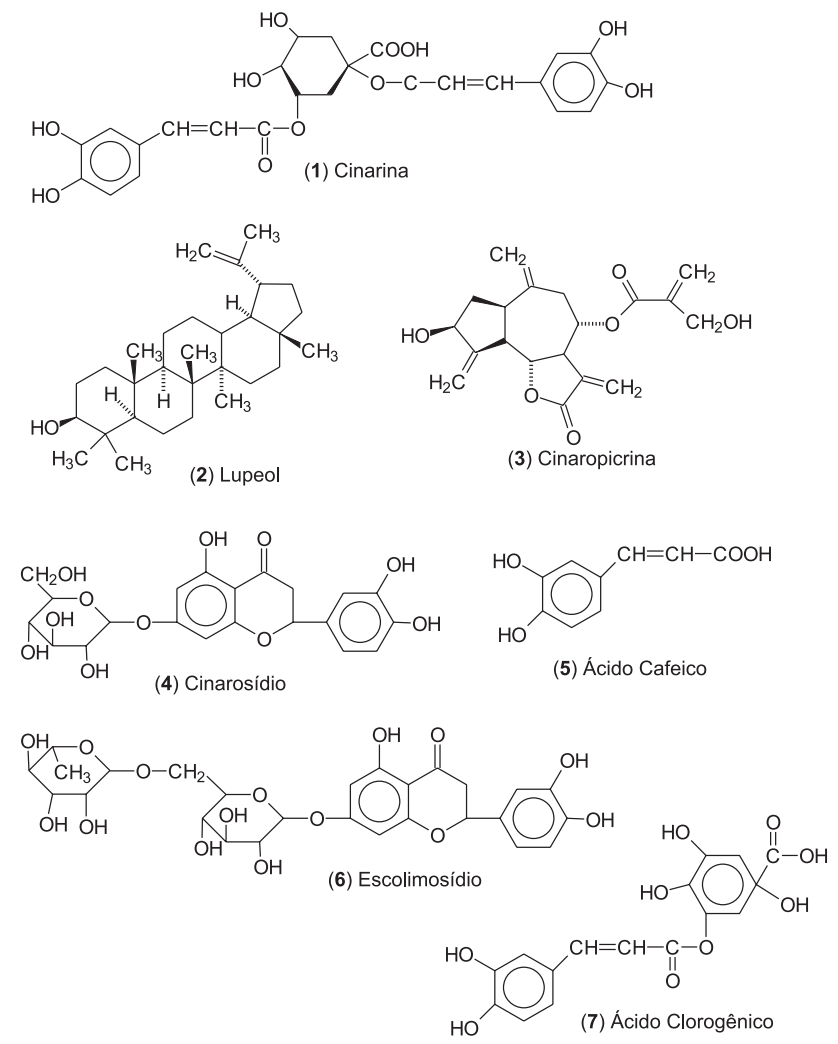

A utilização de bioensaios para o monitoramento da bioatividade de extratos, frações e compostos isolados de plantas tem sido frequentemente incorporada à pesquisa fitoquímica. Dentre estes ensaios biológicos encontra-se o ensaio de citotoxicidade com Artemia salina (BST- Brine Shrimp Test). Inúmeros constituintes bioativos têm sido obtidos de extratos vegetais de plantas conhecidas como 
antitumorais, utilizando-se este teste na monitoração de estudos fitoquímicos ${ }^{32}$. Visando realizar um estudo bio-direcionado, avaliouse o efeito do extrato metanólico bruto (EMB) da alcachofra, das frações hexânica, DCM, AE e butanólica, e também dos compostos 3 e 4 contra Artemia salina. Algumas frações, principalmente as apolares, em diferentes concentrações $(0,5$ a $2 \mathrm{mg} / \mathrm{mL})$ causaram a mortalidade de uma porcentagem significativa da $A$. salina, em particular a fração hexânica, cuja concentração efetiva foi de $358 \pm 72$ $(\mu \mathrm{g} / \mathrm{mL})$, comparável ao controle positivo, sulfato de quinidina. Os compostos puros testados, 3 e 4, não foram ativos até $2 \mathrm{mg} / \mathrm{mL}$.

Quanto à atividade diurética, o extrato metanólico bruto (EMB) demonstrou ter efeito diurético, porém o efeito da fração hexânica, selecionada devido aos melhores resultados obtidos no teste da $A$. salina, foi mais pronunciado. Assim, a fração hexânica mostrou ter efeito aquarético (Tabela 1), pois apresentou um aumento na excreção de água quando comparada ao grupo controle negativo (água), mas não se diferenciou do controle positivo (hidroclorotiazida). Entretanto, esta fração apresentou um perfil distinto da hidroclorotiazida, pois tem maior pico de excreção em 4 h, enquanto que na hidroclorotiazida o maior pico de excreção ocorre nas primeiras duas horas.

A fração hexânica mostrou ter efeito diurético (Tabela 2), já que apresentou uma maior excreção de sódio e potássio quando compa- rada ao grupo controle negativo (água), mas não se diferenciou do controle positivo hidroclorotiazida, sendo que ambas são depletoras de potássio.

O triterpeno lupeol (2), isolado da fração hexânica, não foi citado na literatura como sendo constituinte da alcachofra, sendo este o primeiro relato, podendo ser o composto responsável pelos efeitos citotóxicos e diuréticos encontrados neste estudo. No entanto, as investigações devem ser estendidas para confirmar tal hipótese. Os resultados obtidos permitiram concluir que, embora a cinarina seja o principal constituinte citado na literatura para a planta cultivada em outros países, especialmente na Europa, encontra-se em pouquíssima quantidade na alcachofra cultivada no Brasil. No entanto, novos estudos experimentais são necessários para confirmar tal observação, levando em consideração a sazonalidade, fatores ambientais e processos de preparação dos extratos. Por outro lado, é importante ressaltar a presença da lactona sesquiterpênica cinaropicrina (3) como componente majoritário e do flavonóide cinarosídio (4). O composto $\mathbf{3}$, embora não tenha exibido ação citotóxica no modelo da $A$. salina, é considerado neurotóxico e pode comprometer o uso da alcachofra no Brasil. Isto é altamente preocupante e estimula novos estudos visando um controle de qualidade mais efetivo de fitoterápicos produzidos no país à base de alcachofra.

Tabela 1. Volumes urinários excretados pelos ratos e coletados em gaiolas metabólicas

\begin{tabular}{|c|c|c|c|c|}
\hline Tempo & F. Hexânica (mL) & Ext. Metanólico (mL) & Hidroclorotiazida (mL) & $\mathrm{H}_{2} \mathrm{O}(\mathrm{mL})$ \\
\hline 2 & 1,2 & 0,8 & 3,46 & 0,8 \\
\hline 4 & $3,12 *$ & 1,65 & 1,46 & 0,9 \\
\hline 6 & $1,35^{*}$ & 1,32 & 1,47 & 0,5 \\
\hline 8 & $1,75^{*}$ & 0,9 & 1,72 & 0,4 \\
\hline Volume Final & $5,27 *$ & 3,0 & 7,11 & 2,6 \\
\hline
\end{tabular}

* diferença estatística em relação ao grupo controle negativo (água), $\mathrm{n}=6, \mathrm{p}<0,01$.

Tabela 2. Determinação da excreção de íons

\begin{tabular}{lcccc}
\hline Íons & F. Hexânica $(\mathrm{mEq} / 24 \mathrm{~h})$ & Ext. Metanólico $(\mathrm{mEq} / 24 \mathrm{~h})$ & Hidroclorotiazida $(\mathrm{mEq} / 24 \mathrm{~h})$ & Água $(\mathrm{mEq} / 24 \mathrm{~h})$ \\
\hline Sódio & $33^{*}$ & 6,53 & $49,6^{*}$ & 5,23 \\
Potássio & $64,6^{*}$ & 51,5 & $65,1^{*}$ & 42,0 \\
Cloreto & $2,0^{*}$ & 1,0 & $4,0^{*}$ & 0,85 \\
\hline
\end{tabular}

* diferença estatística em relação ao grupo controle negativo (água), $\mathrm{n}=6, \mathrm{p}<0,01$.

\section{AGRADECIMENTOS}

Os autores agradecem ao apoio financeiro e material vegetal concedidos pelo Laboratório Industrial Vida e Saúde Ltda. (Chapecó$\mathrm{SC})$ e ao $\mathrm{CNPq}$ pelas bolsas recebidas.

\section{REFERÊNCIAS}

1. Cechinel Filho, V.; Yunes, R. A.; Quim. Nova 1998, 21, 99.

2. Kinghorn, A. D.; J. Pharm. Pharmacol. 2001, 53, 135.

3. Yunes, R. A.; Pedrosa, R. C.; Cechinel Filho, V.; Quim. Nova 2001, 24, 147.

4. Yunes, R. A.; Calixto, J. B., eds.; Plantas Medicinais sob a Ótica da Química Medicinal Moderna, Ed. Argos: Chapecó, 2001.

5. Moretto, L. D.; Pharm. Technol. 2000, 4, 46.

6. Capasso, R.; Izzo, A. A.; Pinto, L.; Bifulco, T.; Vitobello, C.; Mascolo, M.; Fitoterapia 2000, 71, S58.

7. Calixto, J. B.; Br. J. Med. Biol. Res. 2000, 33, 179.

8. Freire, S. E.; Urtubey; E.; Acta Farm. Bon. 1999, 18, 192.
9. Newall, C. A.; Anderson, L. A.; Phillipson, D. J.; Herbal Medicines: A Guide for Health-care Professionals, The Pharmaceutical Press: Londres, 1996.

10. Blumenthal, M.; Goldberg, A.; Brinckmann, J., eds.; Herbal Medicine, American Botanical Council: Austin, 2000.

11. Teske, M.; Trentini, A. M. M.; Compêndio de Fitoterapia, 2 ed., Herbarium: Curitiba, 1995

12. Gebhardt, R.; J. Pharm. Exp. Ther. 1998, 386, 1122.

13. Englisch, W.; Beckers, C.; Unkauf, M.; Zinserling, V.; Arzneim. -Forsch. 2000, 50, 260

14. Wenkert, E.; Baddeley, G. U.; Burfitt, I. R.; Moreau, L. N.; Org. Magn. Reson. 1978, 11, 337.

15. Samek, Z.; Holub, M.; Tetrahedron Lett. 1971, 12, 4775.

16. Nawwar, M. A. M.; Mousallamy, A. M.; Barakat, H.; Budrus, J.; Linscheid, M.; Phytochemistry 1989, 28, 3201.

17. Meyer, B. N.; Ferrigini, N. R.; McLaughlin, J. L.; Planta Med. 1982, 45, 31.

18. Kau, S. T.; Keddie, J. R.; Andrews, D. A.; J. Pharmacol. Meth. 1984, 11, 67.

19. Akihisia, T.; Yasukawa, K.; Oinuma, H.; Kasahara, Y.; Yamanouchi, S.; Takido, M.; Kumaki, K.; Tamura, T.; Phytochemistry 1996, 43, 1255. 
20. Malini, M. M.; Lenin, M.; Varalaksmi, P.; Pharmacol. Res. 2000, 41, 413.

21. Nagaraj, M.; Sunitha, S.; Varalakshmi, P.; J. Appl. Toxicol. 2000, 20, 413.

22. Malini, M. M.; Baskar, R.; Varalaksmi, P.; Jpn. J. Med. Sci. Biol. 1995, $48,211$.

23. Moreira, R. R.; Carlos, I. Z.; Vilega, W.; Biol. Pharm. Bull. 2001, 24, 201.

24. Moriarity, D. M.; Huang, J.; Yancey, C.; Zhang, P.; Setzer, W.; Lawton, R. O.; Bates, R. B.; Caldera, S.; Planta Med. 1998, 64, 370.

25. Nikiema, J. B.; Vanhaelen, R.; Vanhaele, M.; Fontaine, J.; Graef, D.; Heenen, M.; Phytother. Res. 2001, 15, 131.

26. Rajic, A.; Kweifio-Okai, G.; Macrides, T.; Sandeman, R. M.; Chandler, D. S.; Polya, G. M.; Planta Med. 2000, 66, 206.

27. Hay, A. J. B.; Hamburger, M.; Hostettmann, K.; Hoult, J. R. S.; Br. J. Pharmacol. 1994, 112, 9.
28. Cho, J. Y.; Park, J.; Yoo, E. S.; Baik. K. ; Jung, J. H.; Lee, J.; Park, M. H; Planta Med. 1998, 64, 594.

29. Cho, J. Y.; Baik, K. U.; Jung, J. H.; Park, M. H.; Eur. J. Pharmacol. 2000, 398, 399.

30. Enriz, R. D.; Rodriguez, A. M.; Jauregui, E. A.; Acta Farm. Bon. 1998, $17,41$.

31. Riopelle, R.J.; Stevens, K.L. Em Bioactive Natural Products; Colegate, S.M.; Molyneux, R. J., eds. ; CRC Press Inc.: Boca Raton/FL, 1993, cap. 19.

32. Siqueira, M. J.; Bonm, D. M.; Pereira, N. F. G.; Quim. Nova 1998, 21, 557. 